\title{
Generation and Control of High Intermittent Heat Load Pattern for Divertor Simulation Studies in GAMMA 10 Tandem Mirror*)
}

\author{
Ryutaro MINAMI, Tsuyoshi IMAI, Tsuyoshi KARIYA, Tomoharu NUMAKURA, Taku EGUCHI, \\ Ryo KAWARASAKI, Kazuhiro NAKAZAWA, Takaki KATO, Fumiya SATO, Hirotaka NANZAI, \\ Makoto UEHARA, Yoichi ENDO, Mafumi HIRATA, Makoto ICHIMURA, Ryuya IKEZOE, \\ Isao KATANUMA, Junko KOHAGURA, Yousuke NAKASHIMA, Kensuke OKI, \\ Mizuki SAKAMOTO, Masayuki YOSHIKAWA and GAMMA 10 Group \\ Plasma Research Center, University of Tsukuba, Tsukuba, Ibaraki 305-8577, Japan
}

(Received 18 November 2013 / Accepted 29 May 2014)

\begin{abstract}
Development of high power gyrotrons and electron cyclotron heating $(\mathrm{ECH})$ systems for the power modulation experiments in GAMMA 10 have been started in order to generate and control the high heat flux and to make the ELM (edge localized mode) like intermittent heat load pattern for divertor simulation studies. ECH for potential formation at plug region $(\mathrm{P}-\mathrm{ECH})$ produces electron flow with high energy along the magnetic filed line. By modulating the ECH power, we can obtain arbitrary pulse heat load patterns. By changing the on/off timing, we can simulate the ELM intermittent heat pulses. The heat flux factor increases almost linearly with ECH power. An intense axial electron flow with energy from hundreds of eV to a few $\mathrm{keV}$ generated by fundamental $\mathrm{P}-\mathrm{ECH}$ is observed. When ECH is turned off, a short burst appears in the end loss ion current due to the axial drain of the confined plasma.
\end{abstract}

(C) 2014 The Japan Society of Plasma Science and Nuclear Fusion Research

Keywords: ECH power modulation, high heat flux, ELM, gyrotron, GAMMA 10 tandem mirror

DOI: $10.1585 / \mathrm{pfr} .9 .3402116$

\section{Introduction}

The new program to simulate the divertor plasma which utilizes advantages of mirror plasma confinement device have been started, in addition to core confinement studies as the mainframe 6 year work plan [1]. Since the boundary plasma physics is the key to sustain the steadystate fusion reactor plasma, the divertor plasma control and plasma wall interaction are urgent issues for ITER and the fusion research. Making use of resources of GAMMA 10 device, we started the GAMMA 10/PDX project, where PDX denotes Potential control and Divertor simulating experiment. The divertor plasma simulator which makes use of high heat flux generated at the open end of the GAMMA 10 is called E-Divertor. It is relevant to the fusion reactor peripheral plasma. This is one of the uniqueness of the GAMMA 10 E-Divertor.

In the GAMMA 10, fundamental ECH at the plug region $(\mathrm{P}-\mathrm{ECH})$ generates the axial ion confining potential $\phi_{\mathrm{c}}$. Experimental observation shows that $\phi_{\mathrm{c}}$ increases with the P-ECH power, and no saturation has been observed as long as the electron density is kept at a certain level. The $\mathrm{P}-\mathrm{ECH}$ drives a portion of the heated electrons into the loss cone and induces an intense axial flow of warm electrons. A portion of the axially flowing electrons is observed as

author'se-mail: minami@prc.tsukuba.ac.jp

*) This article is based on the presentation at the 23rd International Toki Conference (ITC23). end loss electrons. By modulating the ECH power, we can obtain arbitrary pulse heat load patterns. We can expect the ITER level energy density by upgrading ECH to MW level.

Recently, the $1 \mathrm{MW}$, a few seconds, $28 \mathrm{GHz}$ gyrotron for GAMMA 10 and other low B field device ECH source has been developed. Multi-MW and multi-frequency technologies are major issues to challenge for robust and cost effective reactor heating system. Another challenge is EBW (Electron Bernstein Wave) H\&CD which enables EC scheme in lower frequency. This frequency range is required in QUEST of Kyushu University (14 GHz, $28 \mathrm{GHz}$ ), Heliotron $\mathrm{J}$ of Kyoto University $(35 \mathrm{GHz})$ and NSTX-U of PPPL ( $28 \mathrm{GHz}$ ) for EC/EBW H\&CD because of the recent advance in EBW scheme. In recent years, it is applied to the QUEST experiment and has resulted higher EC-driven current than ever [2]. The development of the high power $28 \mathrm{GHz}$ gyrotron is major hardware efforts in GAMMA $10 / \mathrm{PDX}$ project. In the $28 \mathrm{GHz}$ gyrotron development, the output power has been achieved 1.2 MW in the short pulse test, which is new record in this frequency range [3].

In this paper, the empirical scaling of heat flux with $\mathrm{P}-\mathrm{ECH}$ power is studied. The experimental apparatus is given in Section 2, observation of the electron flow is discussed in Section 3 and Section 4 summarizes this work. 


\section{Experimental Apparatus}

The GAMMA 10 is the world largest tandem mirror device (full length of $27 \mathrm{~m}$ ), featuring the MW level high power heating systems. The plasma confinement is achieved by a magnetic mirror configuration as well as positive and negative potentials at the plug/barrier region formed by ECH. The main plasma confined in the central cell of GAMMA 10 is produced by ion cyclotron range of frequency (ICRF) wave. ECH systems $(28 \mathrm{GHz}, 200 \mathrm{~kW}$ at barrier cells and $500 \mathrm{~kW}$ at plug and central cells) are prepared for producing plasma-confining potentials in the plug and barrier regions, and also for direct electron heating in the central cell [4].

Figure 1 shows the P-ECH system and locations of the diagnostic systems used in the preliminary P-ECH modulation experiment to generate the high and ELM-like heat flux.

A gyrotron radiates a quasi-Gaussian beam from a output window. The radiated beam couples to the $\mathrm{HE}_{11}$ mode in the corrugated waveguide of 2.5 inch diameter through the Matching Optics Unit (MOU). The transmission line has two miter bends and it is connected to the GAMMA 10 vacuum vessel. In the vessel, a launcher composed of an open ended corrugated waveguide and two mirrors (M1 and M2) is installed. It radiated the microwave power to the resonance layer as shown in Fig. 1. The microwave beam is obliquely injected into the resonance surface at an angle of $54^{\circ}$. Power density profile on the resonance surface is well fitted to a Gaussian distribution with an e-folded radius of $62 \mathrm{~mm}$. The mirror M2 can be rotated around the horizontal axis and can change the beam direction [5].

The heat flux is measured by the movable calorimeter. This diagnostics instrument is located at $30 \mathrm{~cm}$ downstream from the end-mirror coil $\left(\mathrm{Z}_{\mathrm{EXIT}}=30 \mathrm{~cm}\right)$ and can be inserted from the bottom of the vacuum vessel up to the

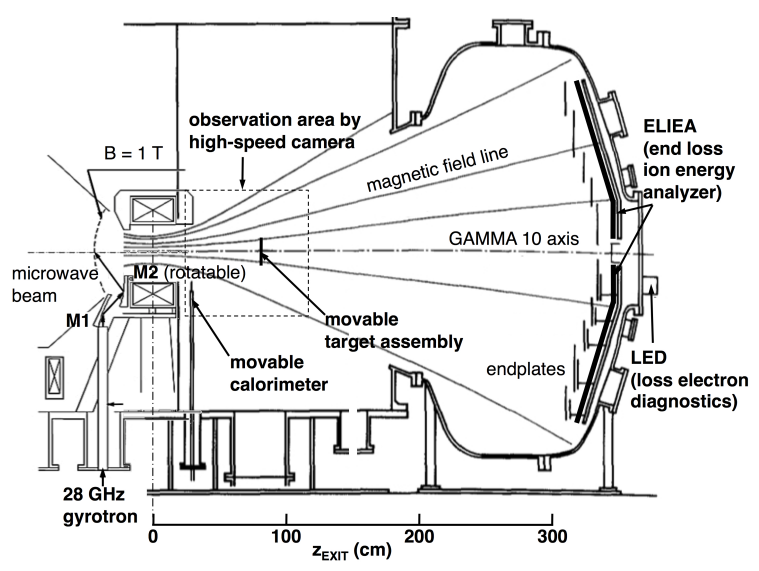

Fig. 1 Cross-section of the plug region and the end region. Microwave power is injected to the $1.0 \mathrm{~T}$ surface from the antenna. The LED is installed behind the innermost endplate. center axis of GAMMA 10. This instrument is also capable of rotation around the shaft, which enables to measure the direction of the plasma flow to the magnetic field line. It is possible to obtain the radial profile of heat flux at $\mathrm{Z}_{\text {EXIT }}=30 \mathrm{~cm}$ by changing the radial position [6].

A set of movable target plates is made to obtain a visible spectroscopic data from the interactions between the plasma and the target materials. The set of target plates is installed at $\mathrm{z}_{\mathrm{EXIT}}=70 \mathrm{~cm}$ through the horizontal observation port. Behavior of the visible emission from the interaction between plasma and the target materials is observed with high-speed cameras. 2-D visible images from cameras are transferred to data analysis PCs through the network [7].

The flux and the energy spectrum of the end loss electrons are measured by a multi-grid energy analyzer (loss electron diagnostics, LED). End loss electrons enter the analyzer through a small hole on an electrically floating end plate that is located in front of the end wall. The collector current of the analyzer corresponds to the electron current flowing into the end plate. The electron repeller voltage of the analyzer is swept and time-resolved current voltage characteristics of the end loss electrons can be obtained in one shot. A sawtooth voltage is applied to the electron repeller grid. The pulse train corresponds to the sawtooth voltage applied the electron repeller grid [8].

The ion currents are measured with spectrometer arrays for end loss ion energy analyses (ELIEAs). Each spectrometer unit in the array has a specific structure with obliquely placed multiple grids with respect to the direction of the ambient plasma confining magnetic fields. This structure has been proposed in order to obtain a precise ion energy spectrum without disturbance from simultaneously incident energetic electrons ranging from a few to several tens of $\mathrm{keV}$ into the array [9].

\section{Experimental Results and Discus- sion}

The plasma experiment for the ECH power modulation is carried out by the use of a plasma discharge after the time of $t=140 \mathrm{~ms}$ (Fig. 2). During P-ECH from $t=150 \mathrm{~ms}$ to $180 \mathrm{~ms}$, the pulse of $\mathrm{ECH}$ power of about $50 \mathrm{~kW}$ for $5 \mathrm{~ms}$ is applied three times by $100 \%, 100 \mathrm{~Hz}$ square wave power modulation (gray region between dotlines in Fig. 2).

The end plate potential is increased during P-ECH injection (Fig. 2(a)). The upward envelope of the pulse train of the end loss electron current during P-ECH injection (Fig. 2 (b)) suggests increase of the population of the loss electron. The pulse train of the electron current is due to sweep of the repeller voltage for energy analysis. Conversely depression of the envelope of the end loss ion current (Fig. 2 (c)) during P-ECH injection suggests decrease of the population of the end loss ion. The time evolution of the visible intensity in the axial direction observed 


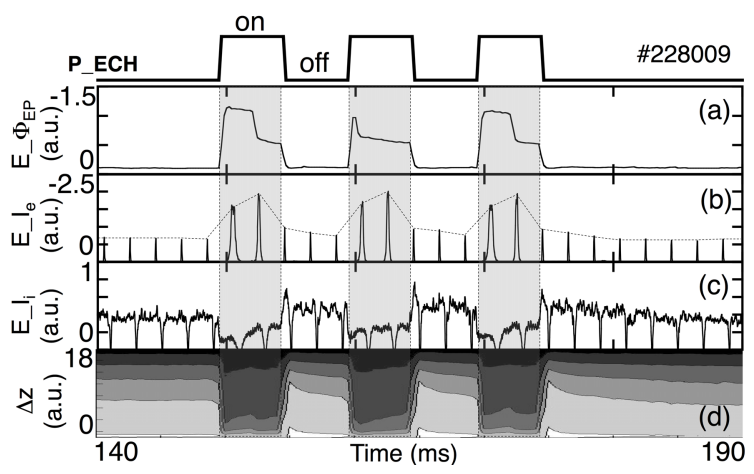

Fig. 2 Temporal evolution of (a) the end plate potential at the east end, the end loss (b) electron current and (c) ion current at the east end and (d) the visible emission from the target plates. The P-ECH power of about $50 \mathrm{~kW}$ is applied from $t=150 \mathrm{~ms}$ to $180 \mathrm{~ms}$. The each pulse is $5 \mathrm{~ms}$ duration with $100 \%, 100 \mathrm{~Hz}$ square wave power modulation.

just front of the center of the target surface is shown in Fig. 2 (d). Black and white colors correspond with low and high intensity level, respectively. It is clearly observed that a rapid reduction of light emission during $\mathrm{ECH}$.

The P-ECH has two functions on electrons by driving two types of velocity space diffusion. The first type of diffusion enhances mirror reflection beyond the plug position, which results in the plug potential. When ECH is applied to the plug region, the end loss ion current decreases owing to the potential reflection of low energy ions (Fig. 2 (c)). The second type of diffusion creates the electron flow (Figs. 2 (a) and 2 (b)). These data indicate that the $\mathrm{P}-\mathrm{ECH}$ is able to control the end loss ion and electron fluxes by the use of power modulation, which can also produce the arbitrary heat load pattern like the various type of the ELM.

The end loss ion current decreases after formation of a confining potential by $\mathrm{ECH}$ and then gradually increase (Fig. 2 (c)). When ECH is turned off, a short burst appears in the end loss ion current due to axial drain of the potentially confined plasma. Consequently, ion flux becomes much higher than the level of before ECH injection.

From these results, we can generate and control arbitrary pulse heat load patterns by modulating the $\mathrm{ECH}$ power. In ECH-on phase, higher electron flux is generated and ion flux is decreased because of confinement potential. In ECH-off phase after power modulation, higher ion flux which is enhanced by potential confinement can be generated. Also, Fig. 2 (d) suggests that the difference of recycling conditions between ECH on/off modulation phase is obtained, which is due to the different interactions between bombarding plasmas and target materials/conditions. A significant change is confirmed in behavior of visible emission from target plates by the differences of end plate potentials, electron and ion energies.

The flux $I_{\mathrm{e}}$ flowing into the collector of the LED

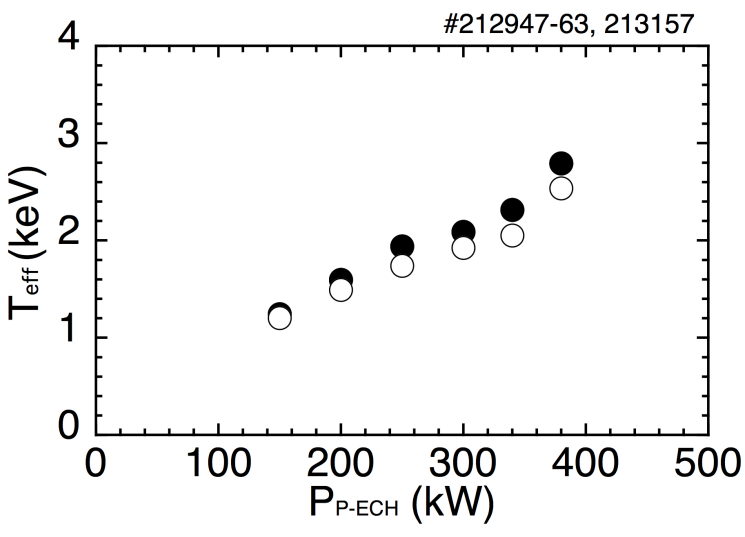

Fig. 3 Effective temperature $T_{\text {eff }}$ plotted as function of the PECH power $P_{\text {P-ECH. }}$. The magnetic field line that passes through the LED corresponds to the radial position of $2.8 \mathrm{~cm}$ in the plug. Closed circles and open circles correspond to west end region and east end region, respectively.

is plotted as a function of the electron repeller voltage $V_{\text {ER }}$. The collector is connected with the machine ground through a low resistance. Both the flux and the mean energy increase with increase in the P-ECH power $P_{\mathrm{P}-\mathrm{ECH}}$. The energy spectra of the end loss electrons cannot be expressed with a single component Maxwellian, but they are well fitted to a two-component Maxwellian:

$$
I_{\mathrm{e}}=I_{\mathrm{L}} \exp \left(-\frac{V_{\mathrm{ER}}+\Phi_{\mathrm{EP}}}{T_{\mathrm{eL}}}\right)+I_{\mathrm{H}} \exp \left(-\frac{V_{\mathrm{ER}}+\Phi_{\mathrm{EP}}}{T_{\mathrm{eH}}}\right),
$$

for $V_{\mathrm{ER}} \geq\left|\Phi_{\mathrm{EP}}\right|\left(\Phi_{\mathrm{EP}}<0\right)$. Here, $T_{\mathrm{eL}}$ denotes the temperature of the bulk component and $T_{\mathrm{eH}}$ is that of the high energy tail component. The value of $I_{\mathrm{e}}$ is constant for $V_{\mathrm{ER}} \leq\left|\Phi_{\mathrm{EP}}\right|$ because the end plate effectively works as an electron repeller. With these parameters an effective temperature $T_{\text {eff }}$ is defined as a measure of the mean energy:

$$
T_{\mathrm{eff}}=(1-\beta) T_{\mathrm{eL}}+\beta T_{\mathrm{eH}},
$$

where $\beta$ is the flux fraction of the $T_{\mathrm{eH}}$ component to the total flux: $\beta=I_{\mathrm{H}} /\left(I_{\mathrm{L}}+I_{\mathrm{H}}\right)$. Here, $\beta$ is evaluated at $V_{\mathrm{ER}}=\left|\Phi_{\mathrm{EP}}\right|$. The dependence of $T_{\text {eff }}$ on $P_{\mathrm{P}-\mathrm{ECH}}$ is presented in Fig. 3. The effective temperature $T_{\text {eff }}$ increases almost linearly with $P_{\mathrm{P}-\mathrm{ECH}}$. This is one of the scalings that express strong effects of the P-ECH.

The investigation of plasma flow from the end-mirror exit of GAMMA 10 is carried out to examine its performance relevant to the divertor simulation studies. A simultaneous measurement of heat and particle fluxes has been carried out by using a movable calorimeter installed at the west end-mirror exit.

By modulating the ECH power, we can obtain arbitrary pulse heat load patterns. By changing the on/off timing, we can simulate the Edge-Localized-Mode (ELM) intermittent heat pulses. The heat flux factor $\left(Q t^{-1 / 2}, Q\right.$ is the ELM deposition energy density and $t$ the ELM deposition time) increases almost linearly with $\mathrm{ECH}$ power. The 
maximum heat flux factor obtained is $0.7 \mathrm{MJ} / \mathrm{m}^{2} \mathrm{~s}^{1 / 2}$ with about $380 \mathrm{~kW} 5 \mathrm{~ms}$ pulse. This is still far lower than that of ITER ELM (mitigated ELMs in ITER is $\sim 22 \mathrm{MJ} / \mathrm{m}^{2} \mathrm{~s}^{1 / 2}$ [10]) but is already to be critical heat load level of long time integrated pulse effect $\left(10^{4}-10^{5}\right.$ cycles $)$.

The effects of intense heat load on tungsten and graphite were estimated by Federici et al. [10]. They showed that lifetime of tungsten plasma facing materials (PFMs) significantly decreased due to melting and evaporation when the ELM energy exceeds $0.4 \mathrm{MJ} / \mathrm{m}^{2}$ assuming the pulse length of $0.1 \mathrm{~ms}$. The severe damage caused by ELM's could be avoided by using recently developed ELM mitigation. Even under the mitigated conditions, however, a change in surface temperature with a frequency of several $\mathrm{Hz}$ causes alternate expansion and contraction, leading to metal fatigue and surface cracking. This phenomena could further reduce a tolerable limit of ELM pulse energy [11]. There are some possibility of contributing to study the effects of ELM-like repetitive pulsed heat load on PFMs by upgrading ECH to MW level. We can expect the ITER level heat flux factor by upgrading ECH to MW level. The $1 \mathrm{MW}$, a few seconds, $28 \mathrm{GHz}$ gyrotron for GAMMA 10 has been already developed. We will be planed to install the new gyrotron for the west side P-ECH.

\section{Summary}

Intermittent high heat pulse simulating the ELM pulse has been successfully generated. The maximum heat flux factor obtained is $0.7 \mathrm{MJ} / \mathrm{m}^{2} \mathrm{~s}^{1 / 2}$ with about $380 \mathrm{~kW} 5 \mathrm{~ms}$ pulse. In ECH-on phase, higher electron flux is generated and ion flux is decreased on account of confinement potential. In ECH-off phase after power modulation, higher ion flux which is enhanced by potential confinement can be generated. It remains a challenge for future research to study the detail electron flow dependence of the target plasma, the radial profiles of microwave power density and so on. According to the increasing and modulating ECH power, it is expected that the heat flux is enhanced and controlled, which enables the ELM resembling heat load pattern. It is possible that the heat flux factor can be increased up to the ITER level with continuous ECH. However, in order to study the effects of not only the energy density itself of ELM in ITER but also the ELM-like repetitive pulsed heat load on PFMs, it remains a challenge for future research to approach to the ITER level by the upgrade and combination of the all heating systems $(\mathrm{ECH}, \mathrm{NBI}$ and ICRF).

\section{Acknowledgments}

The authors would like to appreciate Prof. K. Sakamoto in the Japan Atomic Energy Agency for his collaboration and valuable discussion. This work is partially supported by the grant-in-aid for Scientific Research from Ministry of Education, Science, Sports and Culture of Japan (23560997) and National Institute for Fusion Science (NIFS) Collaborative program (NIFS11KUGM050).

[1] T. Imai et al., Trans. Fusion Sci. Technol. 63, 1T, 8 (2013).

[2] H. Idei et al., US-Japan Workshop Physics of RF Heating of Fusion Plasmas, September 24-26 (2013).

[3] R. Minami et al., Nucl. Fusion 53, 063003 (2013).

[4] T. Kariya et al., Trans. Fusion Sci. Technol. 51, 2T, 397 (2007).

[5] Y. Tatematsu et al., Jpn. J. Appl. Phys. 44, No.9A, 6791 (2005).

[6] Y. Nakashima et al., 23rd IAEA Fusion Energy Conf. FTP/P1-33 (2010).

[7] Y. Nakashima et al., J. Nucl. Mater. 415, S996 (2011).

[8] T. Saito et al., Fusion Eng. Des. 26, 241 (1995).

[9] T. Kuwabara et al., Rev. Sci. Instrum. 65, 936 (1994).

[10] G. Federici et al., J. Nucl. Mater. 290, 260 (2001).

[11] Y. Ueda et al., Fusion Eng. Des. 82, 1904 (2007). 